\title{
Avaliação do conhecimento dos médicos da atenção primária sobre rastreamento de câncer colorretal em um município de Sergipe
}

\author{
Evaluation of knowledge of primary attention physicians on colorectal cancer \\ screening in a city of Sergipe
}

Mayara da Silva Custódio ${ }^{1}$, Anderson Santos dos Anjos ${ }^{1}$, Debora do Nascimento Santos ${ }^{2}$, Fernando Every Belo Xavier ${ }^{3}$, Ana Maria Fantini Silva ${ }^{3}$

\begin{abstract}
RESUMO
Modelo do estudo: Estudo observacional transversal. Objetivo: Avaliar o conhecimento dos médicos prestadores de serviço na Atenção Primária à Saúde do município de Lagarto, Sergipe, sobre o rastreamento das neoplasias colorretais. Metodologia: Estudo quantitativo e qualitativo transversal, realizado com médicos atuantes na Atenção Primária do município de Lagarto, Sergipe. Resultados: Vinte e cinco médicos (92,5\%) responderam ao questionário. A maioria deles (76\%) informou possuir conhecimento necessário para realização do rastreamento do câncer colorretal (CCR). Ao serem questionados, porém, sobre a idade para início do rastreio, apenas $60 \%$ relataram que deve ser aos 50 anos, assim como preconizado pela Organização Mundial de Saúde (OMS). Dezessete (68\%) afirmaram que não há limite de idade para encerrar o rastreamento. Entre os motivos justificados para a não realização do rastreio, 50\% responderam que não o faz porque o paciente não aceita; 33,33\%, por não ter os exames disponíveis no sistema; e 16,66\%, por não possuir conhecimento para conduta. A pesquisa de sangue oculto nas fezes (PSOF) foi escolhida por $92 \%$ dos médicos e a colonoscopia por $60 \%$ deles. Em relação à periodicidade, enquanto $72 \%$ informaram de forma assertiva que a PSOF deve ser realizada anualmente, apenas 36\% relataram que a retossigmoidoscopia deve ser realizada a cada 5 anos. Conclusão: Apesar de a maioria dos médicos inseridos na Atenção Primária do município de Lagarto acreditar possuir o conhecimento necessário para realização do rastreamento do câncer colorretal, observou-se significativa inconformidade em relação às respostas sobre faixa etária alvo e métodos de rastreio quando comparadas ao que a Organização Mundial de Saúde e o Instituto Nacional do Câncer preconizam. A ausência de uma política de saúde pública de prevenção, associada à falta de programas de formação e reciclagem dos profissionais sobre prevenção de câncer colorretal, podem impactar diretamente na manutenção dos altos índices de mortalidade por esta neoplasia.
\end{abstract}

Palavras-chave: Atenção Primária à Saúde. Neoplasias Colorretais. Programas de Rastreamento.

1. Graduado(a) em medicina pela Universidade Federal de Sergipe

2. Graduando em fisioterapia pela Universidade Federal de Sergipe.

3. Professor da Universidade Federal de Sergipe. Departamento de Medicina Lagarto.
CORRESPONDÊNCIA:

Mayara da Silva Custódio

Rua 15 de Novembro, 121

57325-000 - Coité do Nóia/AL - Brasil mayaracustodio001@hotmail.com

Recebido em: 10/07/2018

Aprovado em: 03/04/2019 


\begin{abstract}
Study model: Cross-sectional study. Objective: Evaluating the knowledge of the medical service providers in the Primary Health Care in the city of Lagarto, Sergipe, on the screening of colorectal neoplasias. Methodology: A quantitative and qualitative cross-sectional study carried out with physicians working in Primary Care in the city of Lagarto, Sergipe. Results: Twenty-five physicians $(92.5 \%)$ answered the questionnaire. Most of them (76\%) reported having the necessary knowledge to perform colorectal cancer screening (CRC). When questioned, however, about the age to start screening, only $60 \%$ reported that it should be at the age of 50 , as recommended by the World Health Organization (WHO). Seventeen (68\%) stated that there is no age limit to terminate the screening. Among the reasons for not performing the screening, 50\% answered that it is not performed because the patient does not accept; $33.33 \%$, for not having the exams available in the system; and $16.66 \%$, because they did not have knowledge of the procedure. Fecal occult blood screening (PSOF) was chosen by $92 \%$ of physicians and by colonoscopy for $60 \%$ of them. Regarding periodicity, while $72 \%$ of them reported assertively that PSOF should be performed annually, only $36 \%$ reported that rectosigmoidoscopy should be performed every five years. Conclusion: Although most of the physicians enrolled in Primary Care in the municipality of Lagarto believe that they possess the necessary knowledge to perform the colorectal cancer screening, there was a significant nonconformance regarding the responses on target age group and screening mwethods when compared to what the World Health Organization and the National Cancer Institute advocate. The absence of preventive public health policy, coupled with the lack of training and retraining programs for professionals on the prevention of colorectal cancer, may directly affect the maintenance of high mortality rates due to this neoplasm.
\end{abstract}

Keywords: Primary Health Care. Colorectal Neoplasms. Mass Screening.

\section{INTRODUÇÃO}

O Câncer Colorretal (CCR) compreende as neoplasias malignas que acometem um segmento do cólon, o sigmoide e o reto. É a quarta causa de morte por neoplasia no Brasil. ${ }^{1}$ No sexo masculino, é a quarta neoplasia mais prevalente; no feminino, a terceira. Possui uma história natural da doença bem esclarecida e um período longo de evolução, tornando-o um dos mais sensíveis às medidas preventivas e terapêuticas quando diagnosticado num estágio inicial. ${ }^{2}$ A etiologia do CCR decorre de fatores ambientais e genéticos, com uma maior prevalência de neoplasias esporádicas pela ação dos fatores de risco. ${ }^{3}$

A população ainda possui pouco conhecimento sobre o rastreamento desta neoplasia, o que implica um baixo nível de adesão ao diagnóstico. ${ }^{4}$ Isso culmina em um dos fatores decisivos nas taxas de mortalidade por CCR que é o estadiamento da doença, ou seja, o grau de profundidade e invasão do câncer no momento de seu diagnóstico. ${ }^{5} \mathrm{O}$ rastreamento dessa neoplasia é a principal forma de reduzir a mortalidade. ${ }^{6}$

O Instituto Nacional do Câncer traz alguns exames para realização da detecção precoce desses tumores, sendo preconizados a pesquisa de sangue oculto nas fezes anualmente e o exame endoscópico (colonoscopia e retossigmoidoscopia) com intervalo a cada dez anos. Ainda segundo o INCA, esses exames devem ser solicitados aos indivíduos com sinais e sintomas ou àqueles que se enquadram no grupo de maior risco - mesmo sem apresentar sinais e sintomas. Já a Organização Mundial de Saúde (OMS) preconiza rastreamento em todos aqueles acima de 50 anos nos países com condições de garantir todas as etapas de cuidado ao paciente com este câncer. ${ }^{1} O$ rastreamento deve ser mantido até os 75 anos. Caso haja predisposição familiar ou fatores de risco que aumentem as chances de o indivíduo desenvolver esse tipo de neoplasia, o rastreamento deve ser iniciado precocemente. ${ }^{7}$

Os médicos envolvidos na atenção primária e secundária são, em geral, os principais profissionais a realizarem o diagnóstico de neoplasias, desempenhando, portanto, papel fundamental no diagnóstico precoce dessas doenças. Em um estudo realizado com esses profissionais, observou-se que, dentre os tipos de câncer com menor conhecimento médico sobre o rastreamento, estava o câncer colorretal. O conhecimento sobre as rotinas preconizadas aumenta a eficácia do rastreamento e diminuição da mortalidade. ${ }^{8}$ 


\section{MATERIAIS E MÉTODOS}

Estudo quantitativo e qualitativo transversal, realizado no município de Lagarto, Sergipe. Lagarto fica a 82 quilômetros da capital, Aracaju, e é a maior cidade do interior do Estado. Segundo dados do Instituto Brasileiro de Geografia e Estatística (IBGE), no último censo, em 2010, o município possuía uma população de 94.861 habitantes. $^{9}$

A população estudada foi de vinte e sete médicos que atuavam na Assistência Primária à Saúde da rede pública desta cidade no período analisado. 0 estudo foi realizado entre o período de março a abril do ano de 2018. Ser médico atuante na Atenção Primária à Saúde do município de Lagarto foi o critério de inclusão da pesquisa. Foram excluídos médicos que atendiam exclusivamente a população pediátrica e aqueles que se recusaram a responder o questionário e assinar o Termo de Consentimento Livre e Esclarecido (TCLE). Para avaliação, foi utilizado um questionário intitulado "Neoplasia Colorretal e sua prevenção na Atenção Primária", elaborado pelos autores da pesquisa. O projeto foi encaminhado ao Comitê de Ética da Universidade Federal de Sergipe, aprovado, número do protocolo 2.484 .673 . A participação da pesquisa foi iniciada após esclarecimento de seu objetivo e assinatura do TCLE.

O questionário é composto por doze questões, das quais dez são objetivas e duas subjetivas acerca de Câncer Colorretal, rastreamento, dificuldades encontradas para sua realização, público alvo, exames utilizados e protocolo de referência utilizado pelo profissional médico. Possui, ainda, área aberta para outras informações. A análise das respostas foi baseada nas diretrizes da Organização Mundial de Saúde (OMS). ${ }^{1}$

Os dados foram descritos por frequência simples e percentual. As associações foram avaliadas por meio do teste Exato de Fisher. O software utilizado foi o R Core Team 2018 e o nível de significância adotado foi de $5 \%$.

\section{RESULTADOS}

A pesquisa foi respondida por $92 \%$ dos médicos que atuavam na Atenção Primária no período em questão. Dos médicos atuantes, dois não participaram: um estava afastado de suas atividades; e o segundo se recusou a assinar o TCLE.

Os valores encontrados foram que 19 dos profissionais médicos da rede de Atenção Primária (76\%) julgam ter conhecimento necessário para realização do rastreamento de CCR. Ao serem questionados, no entanto, sobre a idade para início de rastreamento e para seu término, $60 \%$ optou por 50 anos como idade mínima e $68 \%$ marcou que não há idade máxima para sua realização. Setenta e cinco anos foi a segunda opção mais escoIhida para idade máxima com $12 \%$ das respostas. Houve um empate de $8 \%$ para as idades de 85 e 70 anos como máximas.

Entre os $76 \%$ dos profissionais que julgam possuir o conhecimento necessário, $15,78 \%$ não solicitam o rastreamento dessa neoplasia; já três dos que referem não ter conhecimento $(50 \%)$ o realiza.

Seis médicos relataram não realizar o rastreamento de acordo com os seguintes motivos: $16,66 \%$ julgaram não ter conhecimento; 33,33\% alegaram não ter exames disponíveis na rede; e $50 \%$ afirmaram não haver aceitação do paciente para realização dos exames.

A partir do cruzamento de dados entre aqueles que julgam possuir conhecimento necessário para realização do rastreio e a idade selecionada para início do mesmo, verifica-se que $68 \%$ dos médicos que informaram ter conhecimento optaram por 50 anos (Figura 1).

Em relação às medidas utilizadas para realização do rastreamento adequado do CCR, foi possível optar por mais de uma alternativa no questionário (Tabela 1). Além dos exames utilizados, a periodicidade com que eles devem ser solicitados também foi questionada (Tabela 2). 


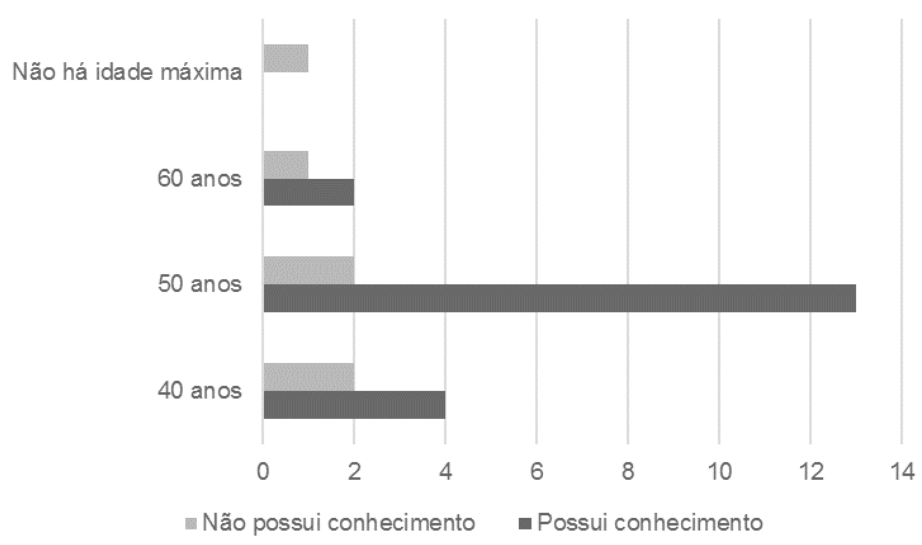

Figura 1: Relação entre médicos que julgam possuir conhecimento para realização do rastreamento e idade de início na Atenção Primária de Lagarto, SE

Fonte: os autores.

\section{Tabela 1}

Exames utilizados para rastreamento de câncer colorretal segundo médicos da Atenção Primária de Lagarto, SE

\begin{tabular}{lc}
\hline Exame & $\mathrm{N}(\%)$ \\
\hline Tomografia Computadorizada & $0(0)$ \\
Pesquisa de Sangue Oculto nas Fezes & $23(92)$ \\
Retossigmoidoscopia & $15(60)$ \\
Ressonância Nuclear Magnética & $3(12)$ \\
Colonoscopia & $16(64)$ \\
Enema Opaco & $2(8)$ \\
\hline
\end{tabular}

$\mathrm{N}$ - Frequência absoluta; \% - frequência percentual

Fonte: os autores.

\section{Tabela 2}

Frequência para realização de exames para rastreamento segundo médicos da Atenção Primária de Lagarto, SE

\begin{tabular}{ccc}
\hline Exame & Frequência & N (\%) \\
\hline $\begin{array}{c}\text { Pesquisa de Sangue } \\
\text { Oculto nas Fezes }\end{array}$ & Anual & $18(72)$ \\
& Bianual & $7(28)$ \\
Retossigmoidoscopia & Anual & $6(24)$ \\
& A cada 5 anos & $9(24)$ \\
& Uma única vez & $4(16)$ \\
& Anual & $6(24)$ \\
Colonoscopia & Bianual & $4(16)$ \\
& A cada 5 anos & $12(48)$ \\
& Uma única vez & $3(12)$ \\
\hline
\end{tabular}

$\mathrm{N}$ - Frequência absoluta; \% - frequência percentual

*Teste Exato de Fisher

Fonte: os autores.
Outro dado relevante observado nos resultados foi que $100 \%$ dos médicos que afirmaram realizar o rastreamento de câncer colorretal em sua prática clínica utilizam a Pesquisa de Sangue Oculta nas fezes como exame de escolha $(p=0,05)$. A retossigmoidoscopia foi escolhida por $68 \%$ dos que realizam e a colonoscopia por $63 \%$. A Ressonância Nuclear Magnética e o Enema Opaco foram escolhidos por $10,53 \%$ dos entrevistados (Tabela 3 ).

\section{Tabela 3}

Relação entre médicos que solicitam rastreamento em sua conduta e o tipo de exame utilizado.

\begin{tabular}{|c|c|c|c|}
\hline \multirow[t]{2}{*}{ Exames } & \multicolumn{2}{|c|}{$\begin{array}{l}\text { Em sua prática } \\
\text { clínica você solicita } \\
\text { rastreamento para } \\
\text { colorretal? }\end{array}$} & \multirow[t]{2}{*}{ p-valor* } \\
\hline & $\begin{array}{l}\text { Sim } \\
N(\%)\end{array}$ & $\begin{array}{l}\text { Não } \\
\text { N (\%) }\end{array}$ & \\
\hline $\begin{array}{l}\text { Tomografia } \\
\text { Computadorizada }\end{array}$ & $\begin{array}{c}0 \\
(0)\end{array}$ & $\begin{array}{c}0 \\
(0)\end{array}$ & - \\
\hline $\begin{array}{l}\text { Pesquisa de Sangue } \\
\text { Oculto nas fezes }\end{array}$ & $\begin{array}{c}19 \\
(100)\end{array}$ & $\begin{array}{c}4 \\
(66,67)\end{array}$ & 0,050 \\
\hline Retossigmoidoscopia & $\begin{array}{c}13 \\
(68,42)\end{array}$ & $\begin{array}{c}2 \\
(33,33)\end{array}$ & 0,175 \\
\hline $\begin{array}{l}\text { Ressonância Nuclear } \\
\text { Magnética }\end{array}$ & $\begin{array}{c}2 \\
(10,53)\end{array}$ & $\begin{array}{c}1 \\
(16,67)\end{array}$ & 1,000 \\
\hline Colonoscopia & $\begin{array}{c}12 \\
(63,16)\end{array}$ & $\begin{array}{c}4 \\
(66,67)\end{array}$ & 1,000 \\
\hline Enema opaco & $\begin{array}{c}2 \\
(10,53)\end{array}$ & $\begin{array}{c}0 \\
(0)\end{array}$ & 1,000 \\
\hline \multicolumn{4}{|c|}{$\mathrm{N}$ - frequência observada; \% - frequência percentual } \\
\hline \multicolumn{4}{|l|}{ *Teste Exato de Fisher } \\
\hline \multicolumn{4}{|l|}{ Fonte: os autores. } \\
\hline
\end{tabular}


O estudo analisou, também, os grupos de risco para o surgimento de CCR - os entrevistados podiam optar por mais de uma alternativa. Histórico prévio ou familiar de neoplasia ficou com um total de $92 \%$; portadores de Doença Inflamatória Intestinal (DII), com $80 \%$; tabagista, $64 \%$; pessoas sintomáticas, $44 \%$; e doença hemorroidária, 32\% da estatística.

O questionamento aberto foi sobre as principais dificuldades que o profissional encontrava para realização do rastreamento e a última foi para "outros comentários". Dezessete dos médicos citaram a falta de exames disponíveis no sistema como a principal dificuldade. A falta de informação do paciente sobre a doença e a importância da realização do exame foram mencionadas por oito médicos. Um médico mencionou a qualidade dos laboratórios para realização da Pesquisa de Sangue Oculto nas fezes como dificuldade. A falta de serviço especializado para encaminhamento dos pacientes e a falta de protocolo referente ao tema foram também citadas por uma minoria dos entrevistados. Não houve respostas para a pergunta de "outros comentários".

\section{DISCUSSÃO}

O estudo abordou o conhecimento e a atitude dos médicos da Atenção Primária à Saúde de Lagarto, Sergipe, com amostra satisfatória para pesquisa.

o Câncer Colorretal (CCR) é atualmente uma das mais prevalentes causas de morte por câncer. Isso o torna um importante problema de saúde, pois, a cada ano no mundo são diagnosticados cerca de um milhão de novos casos, sendo que, desse número, metade vai a óbito. ${ }^{10}$

No Estado de Sergipe, o CCR está classificado como quinto mais incidente. Estimaram-se 70 casos novos em 100 mil habitantes para o gênero masculino; já para o gênero feminino, 100 novos casos em 100 mil habitantes. Os índices de mortalidade nacionais estão em 15.415 , sendo 7.387 homens e 8.024 mulheres. $^{2}$

Já foi demonstrado que o fator decisivo nas altas taxas de mortalidade por CCR está no estadiamento avançado no momento de seu diagnóstico. ${ }^{5} \mathrm{O}$ rastreamento adequado dessa neoplasia é a principal forma de reduzir as elevadas taxas de mortalidade. ${ }^{6}$ Para que isso seja feito, é necessário conhecimento adequado dos médicos sobre os protocolos que determinam o público alvo, grupos de alto risco e exames a serem solicitados.

Dos médicos participantes da pesquisa, $76 \%$ afirmaram ter conhecimento necessário para realizar o rastreio dessa neoplasia. Esse resultado aproxima-se do valor de $80 \%$ encontrado em 2011 por Souza et al., que estudaram a realização de rastreamento para CCR com médicos que trabalhavam no Hospital da Universidade Luterana do Brasil e em unidades básicas de saúde no Rio Grande do Sul. ${ }^{11}$

Entre os motivos pelos quais os médicos não realizam o rastreamento em sua conduta, $50 \%$ dos entrevistados responderam que não há aceitação do paciente para sua realização. Esse dado vai ao encontro dos $82,86 \%$ encontrados em 2004 por Tucunduva et al., quando entrevistaram médicos não oncologistas da escola de medicina de São Paulo, que afirmaram ter como barreira e fator de grande importância a falta de agentes educadores em saúde para população. ${ }^{8}$

Tucunduva et al. demonstraram ainda que $77,14 \%$ justificaram a falta de conhecimento médico como de grande importância. ${ }^{8}$ No presente estudo, no entanto, apenas $16,6 \%$ da amostra referiram que a não realização do rastreamento está ligada à falta de conhecimento sobre o tema. Os 33,33\% restantes alegaram o fato de não ter exames médicos disponíveis na rede do Sistema Único de Saúde (SUS).

Analisando as respostas de acordo com o recomendado pela Organização Mundial de Saúde (OMS) - que o rastreamento deve ser realizado a partir dos 50 anos para todos os indivíduos ${ }^{1}-$, o estudo mostrou que $60 \%$ dos médicos estão em concordância com recomendação supracitada. A mesma porcentagem foi demonstrada em outro estudo realizado em 2011. ${ }^{11}$

Segundo REX et al., 2017, o rastreamento deve ser feito com Pesquisa de Sangue Oculto nas Fezes (PSOF) anualmente e com colonoscopia a cada 10 anos. ${ }^{12}$ Ao questionar sobre quais exames eram utilizados para o rastreamento, 92\% dos participantes optaram pela PSOF, seguida pela colonoscopia escolhida por $64 \%$ dos médicos. Isso difere do estudo realizado por Souza et al. em 2011 que mostrou uma prevalência de colonoscopia e PSOF em $83 \%$ e $73 \%$ dos 
casos, respectivamente, como exame de escolha para o rastreio.

O Caderno de Atenção Primária n.o 29, publicado no ano de 2010, que serve de orientação para os profissionais de saúde, traz menção ao câncer colorretal e seu rastreamento. Apesar da recomendação de Pesquisa de Sangue Oculto nas Fezes em pacientes de 50 a 75 anos, o caderno não sugere unicamente esse tipo de rastreamento, pois, o número de falso-positivo chega a $80 \%$ dos achados. ${ }^{13}$

Silva et al., em 2011, demonstraram que a maioria dos médicos possuía um conhecimento limitado sobre diretrizes e recomendações do câncer colorretal, com valores inferiores a $11 \%$ de conformidades, gerando condutas deficientes. ${ }^{14} \mathrm{O}$ mesmo foi possível ser visualizado no presente estudo em que a idade para início do rastreio, os exames utilizados e a periodicidade que devem ser realizados, em sua maioria, não condiziam com o recomendado. Tucunduva et al., em 2004, também demonstraram essa mesma deficiência, ou seja, em relação ao CCR, que nenhum médico informou conduta adequada para o rastreamento populacional. ${ }^{4}$

As limitações metodológicas deste estudo estão no número reduzido de profissionais na Atenção Básica do município em tela. Apesar da pequena amostra, foi possível demonstrar que os resultados encontrados se assemelham aos de outros estudos, sugerindo que há uma falha real em relação ao conhecimento acerca da prevenção e rastreamento do câncer colorretal.

\section{CONCLUSÃO}

Este estudo demonstrou que, apesar de a maioria dos profissionais médicos inseridos na Atenção Primária afirmar possuir o conhecimento necessário para realização do rastreamento do câncer colorretal, tais índices não foram observados após a análise de dados. Esse fato pode contribuir para a manutenção dos altos índices de mortalidade por essa neoplasia.

Dificuldades como não aceitação do paciente, não conhecimento médico e não disponibilidade dos exames na rede pública de saúde são fatores decisivos em suas condutas. ${ }^{13}$
Os resultados deste estudo apontam para necessidade de protocolos e divulgação desse rastreio para a população médica e usuários de modo a minimizar essa problemática. Efetivar a prevenção secundária deve ser o objetivo do sistema para reduzir mortalidade e gastos com prevenção terciária e quaternária.

\section{Declaração de Conflitos de Interesse}

Nada a declarar.

\section{REFERÊNCIAS}

1. Instituto Nacional de Câncer [homepage na Internet]. Colorretal; 2018 [acesso em 18 jun 2018]. Disponível em: http://www2.inca.gov.br/wps/wcm/connect/tiposdecancer/site/home/colorretal/definicao

2. Instituto Nacional do Câncer [homepage na internet]. Rio de Janeiro: Estimativa 2016: Incidência de Câncer no Brasil; 2015 [acesso em 11 jun 2019]. Disponível em: http://santacasadermatoazulay.com.br/wp-content/uploads/2017/06/estimativa-2016-v11.pdf

3. Leser SM, Soares E de A. Aspectos nutricionais e atividade física na prevenção do câncer colorretal. Nutrire: rev. Soc. Bras. Alim. Nutr. 2001 [acesso em 3 ago 2017];21:121-145. Disponível em: http://sban.cloudpainel.com.br/files/revistas_publicacoes/20.pdf

4. Santos TP dos, Carvalho LP de, Souza ECR e, Lise M. Conhecimento dos usuários do serviço público de saúde sobre câncer colorretal e sua prevenção. AMRIGS. 2013 [acesso em 3 ago 2017];57:31-38. Disponível em: https://pdfs.semanticscholar.org/dece/782298cee48b6673af0c6468867ba2755305.pdf

5. Torres Neto J da R, Teixeira FR, Prudente $A C L$, Silvino $C J$, Arciere JS, Vieira Filo MC. Estudo demográfico do câncer de cólon e reto no estado de Sergipe. Rev bras coloproct. 2008 [acesso em 18 jun 2018];28(2):215-222. Disponível em: http://www.scielo.br/pdf/rbc/v28n2/10.pdf

6. Inadomi JM. Screening for Colorectal Neoplasia. N Engl J Med. 2017 [acesso em 3 ago 2017];376:149-156. doi: 10.1056/NEJMcp 1512286

7. Ferrari CLS. A prevenção do câncer ao alcance de todos. Guia prático para prevenção do câncer [internet]. 2016 [acesso em 24 jun 2018]. Disponível em: https://www. sboc.org.br/images/downloads/pacientes/SBOC_Prevencao-do_Cancer.pdf

8. Tucunduva LTC de M, Sá VHLC de, Koshimura ET, Prudente FVB, Santos AF dos, Samano EST, et al. Estudo da atitude e do conhecimento dos médicos não oncologistas em relação às medidas de prevenção e rastre- 
amento do câncer. Rev Assoc Med Bras. 2004 [acesso em 18 jun 2018];50(3):257-262. Disponível em: http://www.scielo.br/scielo.php?script=sci_arttext\&pi$d=$ S0104-42302004000300030\&Ing=en

9. Instituto Brasileiro de Geografia e Estatística. [homepage na internet]. Brasil / Sergipe / Lagarto: Panorama; 2018 [acesso em 18 jun 2018]. Disponível em: https:// cidades.ibge.gov.br/brasil/se/lagarto/panorama

10. Andrade LNP de, Soares A. Pesquisa de Sangue Oculto nas Fezes e o Diagnóstico de Câncer Colorretal. Simpósio de TCC e Seminário de IC. 2016 [acesso em 18 jun 2018]. Disponível em: http://nippromove.hospedagemdesites.ws/anais_simposio/arquivos_up/documentos/ artigos/96bb16bc249b151b7896fcb99a34ce71.pdf

11. Souza E da CR, Lise M, Santos TP dos, Carvalho LP de. Knowledge and practice of physicians regarding colorectal cancer screening. J. Coloproctol. (Rio J.). 2012 [acesso em 16 jun 2016];32():385-394. Disponível em: http://www.scielo.br/scielo.php?script=sci_arttext\&pid=S2237-93632012000400005
12. Rex DK, Boland CR, Dominitz JA, Giardiello FM, Johnson DA, Kaltenbach $T$, et al. Colorectal Cancer Screening: Recommendations for Physicians and Patients From the U.S. Multi-Society Task Force on Colorectal Cancer. Gastroenterology. 2017 [acesso em 10 jun 2018];153:307-323. Disponível em: http://sci-hub.tw/10.1053/j.gastro.2017.05.013

13. Ministério da Saúde. Caderno de Atenção Primária: Rastreamento [internet]. Brasilia: Ministério da Saúde; 2010 [acesso em 3 de ago 2017]. Disponível em: http://189.28.128.100/dab/docs/publicacoes/cadernos_ab/abcad29.pdf

14. Silva LMC da, Fonseca AJ da, Ferreira LP, Dalla-Benetta AC, Navarro C. Atitude e Conhecimento de Médicos da Estratégia Saúde da Família sobre Prevenção e rastreamento do Câncer. Rev Bras Cancerologia. 2011 [acesso em 16 jun 2018];57(4):525-534. Disponível em: http:// www1.inca.gov.br/rbc/n_57/v04/pdf/09_artigo_atitude_e_conhecimento_medicos_estrategia_saude_familia_sobre_prevencao_rastreamento_cancer.pdf 